\title{
Análise crítica de estudos brasileiros sobre deficiência de vitamina A no grupo materno-infantil
}

\author{
Critical analysis of Brazilian studies about vitamin A deficiency in maternal-child group
}

\author{
Andréa Ramalho' ${ }^{1}$ Patrícia Padilha², Cláudia Saunders ${ }^{3}$
}

\section{RESUMO}

Objetivo: Apresentar uma análise crítica de estudos da literatura sobre a deficiência de vitamina A (DVA) no Brasil, segundo o indicador bioquímico retinol sérico, considerandose o impacto negativo da DVA na saúde reprodutiva e no desenvolvimento infantil.

Fontes de dados: os bancos de dados Medline e BVS, no período de 1994 a 2007, foram rastreados com a combinação de descritores: "vitamin A deficiency", "Brazil", "pregnant", "children" e "retinol serum level". A inclusão dos artigos foi realizada com base em critérios de elegibilidade referentes à população, desenho do estudo e idioma, enfatizando-se estudos com gestantes, puérperas, nutrizes, lactentes e pré-escolares que não apresentassem doenças crônicas nãotransmissíveis ou infecciosas.

Síntese dos dados: dos 27 artigos selecionados, 25 eram estudos transversais, um era caso-controle e um estudo longitudinal. Identificou-se que $71 \%$ e $25 \%$ dos trabalhos com gestantes e crianças, respectivamente, adotaram valores de retinol sérico $<1,05 \mu \mathrm{mol} / \mathrm{L}$ como ponto de corte. Todos os estudos apontaram a DVA como um problema de proporção preocupante. Reconhece-se a emergente inserção dos adolescentes no grupo de risco potencial para DVA e da antropometria como variável independente para tal deficiência nutricional.

Conclusões: Aponta-se uma necessidade de incluir estratégias de intervenção adicionais à suplementação, reavaliar pontos de corte na interpretação do diagnóstico da DVA e refletir sobre a pertinência da inclusão de

Instituição: Instituto de Nutrição Josué de Castro (INJC) da Universidade Federal do Rio de Janeiro (UFRJ), Rio de Janeiro, RJ, Brasil

${ }^{1}$ Doutora em Ciências pela Escola Nacional de Saúde Pública (ENSP) da Fundação Oswaldo Cruz (Fiocruz), professora titular do Departamento de Nutrição Social e Aplicada, coordenadora do Núcleo de Pesquisa em Micronutrientes (NPqM) do INJC da UFRJ, Rio de Janeiro, RJ, Brasil

${ }^{2}$ Nutricionista do Instituto de Puericultura e Pediatria Martagão Gesteira da UFRJ, doutoranda do Programa de Pós-graduação em Nutrição do INJC da UFRJ, pesquisadora do Grupo de Pesquisa em Saúde Materna e Infantil (GPSMI) do NPqM do INJC da UFRJ, Rio de Janeiro, RJ, Brasil

${ }^{3}$ Doutora em Ciências pela ENSP da Fiocruz, professora adjunta do Departamento de Nutrição e Dietética do INJC da UFRJ e da Maternidade Escola da UFRJ, coordenadora do GPSMI do NPqM do INJC da UFRJ, Rio de Janeiro, RJ, Brasil novas áreas e grupos com risco potencial para DVA em programas de intervenção.

Palavras-chave: deficiência de vitamina A; epidemiologia; nutrição; indicadores.

\section{ABSTRACT}

Objective: To critically review the literature regarding vitamin A deficiency (VAD) in Brazil, according to biochemical indicators, considering that VAD has a negative impact upon reproductive health and child development.

Data source: Medline and BVS databases were searched from 1994 to 2007, using the combination of the following terms: "vitamin A deficiency", "Brazil", "pregnant", "children" and "retinol serum level". Inclusion criteria were based on characteristics of the studied population, study design and language, with emphasis on studies with the following subjects: pregnant, postpartum and nursing women, infants and preschool children without chronic or infectious diseases.

Data synthesis: Among the 27 selected studies, 25 were cross-sectional, one was a case-control, and one was a cohort study. $71 \%$ and $25 \%$ of the studies with pregnant women and with children, respectively, adopted retinol serum level $<1.05 \mu \mathrm{mol} / \mathrm{L}$ as the cut-off value for VAD. All the studies pointed VAD as a health problem of major concern. The need of including adolescents as a risk group of VAD is recognized. Anthropometry should be

Endereço para correspondência:

Andréa Ramalho

INJC do Centro de Ciências da Saúde da UFRJ

Bloco J, $2^{\circ}$ andar - Ilha do Fundão

CEP 21944970 - Rio de Janeiro/RJ

E-mail: aramalho@rionet.com.br

Recebido em: 13/4/2008

Aprovado em: 4/8/2008 
considered as an independent variable for this nutritional deficiency.

Conclusions: The findings of this review show the need of aditional intervention strategies regarding supplementation of vitamin A. VAD should be secuched not only in risk areas, but throughout the country, in several potential risk groups. It is important to reevaluate cut-off points in order to better diagnose vitamin A deficiency.

Key-words: vitamin A deficiency; epidemiology; nutrition; indicators.

\section{Introdução}

As deficiências nutricionais, em especial de micronutrientes, têm merecido destaque e atenção dos pesquisadores pelo número crescente de evidências na literatura que comprovam seu impacto na morbimortalidade dos grupos de maior vulnerabilidade: as mulheres em idade fértil e as crianças. Nesse escopo, encontra-se a deficiência da vitamina A (DVA), cuja relevância como problema de Saúde Pública é inquestionável ${ }^{(1-3)}$.

Atualmente, sabe-se que mesmo a deficiência da vitamina A subclínica (quando estão ausentes os sinais de xeroftalmia) intensifica a gravidade de enfermidades como diarréia e outros processos infecciosos, podendo provocar quadros de imunodeficiência de origem exclusivamente nutricional ${ }^{(1-4)}$. Calcula-se que, a cada minuto, morra uma criança de causa direta ou indiretamente atribuível à deficiência da vitamina A e que, diariamente, muitas mulheres falecem em decorrência de problemas evitáveis relacionados à gravidez ou ao parto, e que podem ser agravados pela deficiência da vitamina $A^{(5)}$.

Na década de 1980, as atividades de controle da deficiência da vitamina A tiveram início no Brasil, sendo adotada a estratégia de suplementar com doses maciças de vitamina A crianças em idade pré-escolar de áreas específicas do país. Áreas essas consideradas de alto risco para o desenvolvimento da deficiência desse micronutriente por características geográficas, climáticas e econômicas ${ }^{(6)}$. Em 1994, o Ministério da Saúde (MS) criou o Programa Nacional de Controle das Deficiências de Vitamina A no âmbito do extinto Instituto de Alimentação e Nutrição (INAN) ${ }^{(6)}$.

Em 1998, o Programa de Alimentação e Nutrição, juntamente com a Divisão de Vacinas e Imunização e o Programa Ampliado de Imunização da Organização Pan-
Americana de Saúde (OPAS) desenharam um projeto que previa a suplementação com vitamina $A$ na região das Américas para lactentes e pré-escolares (de seis a 59 meses), com periodicidade de quatro/seis meses. As puérperas no período até seis semanas pós-parto também deveriam receber doses de vitamina A. Além do Brasil, onde as áreas priorizadas foram aquelas consideradas como 'bolsões endêmicos' da deficiência da vitamina $A$, outros países também participaram do programa: Bolívia, Equador, Nicarágua, Peru, e República Dominicana ${ }^{(7)}$.

Recentemente, o MS instituiu, através da Portaria 729 de 13 de maio de 2005, o Programa Nacional de Suplementação de Vitamina A, intitulado 'Vitamina A Mais'(8). Sua finalidade é reduzir e controlar a deficiência da vitamina A em crianças de 6 a 59 meses de idade e mulheres no pós-parto imediato que residam nas áreas consideradas de risco para a deficiência (Nordeste, região norte de Minas Gerais, Vale do Jequitinhonha, Vale do Murici e Vale do Ribeira em São Paulo). Contudo, o MS reconhece que subsistem dúvidas acerca da extensão e magnitude desta deficiência nas demais regiões do país. A título de exemplo, na Região Sudeste, mais precisamente nas cidades do Rio de Janeiro e São Paulo, estudos recentes documentaram elevada prevalência de deficiência da vitamina $\mathrm{A}^{(9-17)}$. O programa prevê para mulheres no pós-parto imediato a suplementação com dose única de 200.000UI de vitamina A ainda na maternidade e, para crianças com idade entre 6 e 59 meses, suplementação a cada seis meses, com distribuição da dose de acordo com a idade ${ }^{(8)}$. Além dessas medidas, registradas na nova Caderneta de Saúde da Criança ${ }^{(18)}$, recomenda-se, como estratégias de intervenção adicionais para o combate à deficiência da vitamina A, promoção do aleitamento materno e da alimentação saudável, com estímulo ao consumo de alimentos fontes desta vitamina pela população.

O presente artigo objetiva apresentar uma análise crítica de estudos disponíveis na literatura sobre a deficiência da vitamina A no Brasil a partir da implantação do Programa de Suplementação de vitamina A segundo o indicador bioquímico retinol sérico. Leva-se em consideração o fato de que tal deficiência tem impacto negativo na saúde reprodutiva e no desenvolvimento infantil, o que contribui para o aumento dos índices de morbimortalidade no binômio mãe-filho. Tal levantamento pode subsidiar o planejamento de novos estudos e de políticas públicas direcionadas ao combate das carências nutricionais específicas. 


\section{Métodos}

Trata-se de uma revisão da literatura com seleção criteriosa de artigos a partir de estratégia de busca definida e passível de reprodução sobre a prevalência da deficiência da vitamina A no Brasil. Enfatizou-se o grupo materno-infantil, por meio dos indicadores bioquímicos (níveis de retinol sérico), a partir da implantação do Programa de Suplementação de Vitamina A.

Foram incluídos trabalhos realizados com gestantes, puérperas e nutrizes (adultas e adolescentes), lactentes, pré-escolares e escolares que não apresentassem doenças crônicas não-transmissíveis ou infecciosas, com exceção da deficiência de micronutrientes. Foram considerados estudos com a suplementação somente de vitamina $\mathrm{A}$ ou com essa vitamina associada à suplementação de ferro e ácido fólico.

Os artigos foram identificados por meio de busca na base de dados Medline versão PubMed (http://www.pubmed.gov) e Biblioteca Virtual em Saúde (BVS) (http://www.bireme.br). Os seguintes descritores foram utilizados na busca: vitamin A deficiency, retinol serum level, Brazil, pregnant, children, post partum women.

Os resumos de artigos selecionados foram lidos por três avaliadores que decidiram sobre a inclusão dos mesmos com base em critérios de elegibilidade referentes à população de estudo, aos desenhos das pesquisas (transversais, caso-controle, e longitudinal) e ao idioma (português, inglês ou espanhol). Cada avaliador, de modo independente, decidia por "inclusão" ou "exclusão". Os resultados discrepantes foram avaliados por consenso posterior entre os avaliadores.

A busca limitou-se aos artigos publicados no período de 1994 (início do Programa de Suplementação de Vitamina A) a 2007. Esses critérios de inclusão foram previamente estabelecidos com o objetivo de definir claramente a adequação da literatura encontrada para esse estudo de revisão. Foram excluídos da revisão artigos publicados em idiomas além dos acima referidos, estudos com animais, pesquisas in vitro, revisões, ensaios com a utilização de outros indicadores do estado nutricional de vitamina $A$, estudos não conclusivos e aqueles com metodologia não definida.

A seleção dos artigos identificados nas bases de dados realizou-se em três fases. $\mathrm{Na}$ etapa inicial houve avaliação preliminar da relevância do estudo para uma revisão crítica, de acordo com os critérios de inclusão e exclusão pré-deter- minados. Na segunda, foi realizada a apreciação crítica dos estudos pelos revisores. Os artigos considerados elegíveis foram comparados em relação a: região do país, desenho do estudo, características da população, critério diagnóstico da deficiência da vitamina $\mathrm{A}$ e resultados observados nos grupos de estudo.

Definiu-se como desfecho de interesse primário a deficiência da vitamina A e, como desfechos secundários, a concentração de retinol sérico materno ou infantil, a classificação de deficiência da vitamina A subclínica de acordo com o teste de resposta relativa à dose (RDR) e a resposta sérica de 30 dias relativa à dose $(+$ S30DR) no grupo materno-infantil.

As concentrações de retinol são apresentadas em $\mu \mathrm{mol} / \mathrm{L}$. Para estudos que apresentaram os dados de retinol em $\mu \mathrm{g} /$ dL, foi realizada a conversão necessária. Os valores de concentração de vitamina A são apresentados em percentual de inadequação conforme constam nos artigos originais.

\section{Resultados}

A pesquisa inicial realizada nas bases de dados eletrônicas identificou 300 referências. Após análise criteriosa dos artigos identificados, exclusão dos repetidos e leitura dos resumos, foi feita uma nova seleção com base nos critérios de inclusão, sendo totalizados 27 trabalhos, dos quais 20 realizados com recém-nascidos, pré-escolares e/ ou com escolares e sete com gestantes ou puérperas. Dos 27 artigos selecionados, 25 eram estudos transversais, um caso-controle e um longitudinal.

Verificou-se que, em 17 (63\%) estudos, foi adotado o ponto de corte para níveis séricos de retinol $<0,70 \mu \mathrm{mol} / \mathrm{L}$ ou $0,35 \mu \mathrm{mol} / \mathrm{L}$ para definir a deficiência da vitamina A. O ponto de corte de retinol sérico $<1,05 \mu \mathrm{mol} / \mathrm{L}$ foi adotado em $71 \%(n=5)$ e $25 \%(n=5)$ dos estudos realizados com gestantes e com recém-nascidos, pré-escolares e/ou escolares, respectivamente.

Nos artigos analisados, a área do país mais estudada foi a região sudeste, contemplando $86 \%(\mathrm{n}=6)$ dos trabalhos com gestantes ou puérperas e $60 \%(n=12)$ daqueles com recém-nascidos, pré-escolares e escolares. O grupo mais investigado foi o de recém-nascidos, pré-escolares e escolares ( $74 \% ; n=20)$, com destaque para os pré-escolares em $41 \%$ $(\mathrm{n}=11)$ dos estudos.

As Tabelas 1 e 2 trazem os dados da prevalência de deficiência da vitamina $\mathrm{A}$, a área estudada, o tipo de estudo, o tamanho amostral e o ponto de corte adotado. 
Tabela 1 - Prevalência da deficiência de vitamina A em gestantes e puérperas, em diferentes regiões do Brasil

\begin{tabular}{|c|c|c|c|c|}
\hline Ano & Local do estudo & Tipo de estudo & Grupo populacional & Prevalência (\%) \\
\hline $1998^{(13)}$ & Rio de Janeiro, RJ & Transversal & Puérperas & $23,2^{(a)}$ \\
\hline \multirow[t]{2}{*}{$1999^{(33)}$} & SP - fora da capital & Transversal & Puérperas & $10,2^{(a)}$ \\
\hline & & & & $1,3^{(b)}$ \\
\hline $2000^{(10)}$ & Rio de Janeiro, RJ & Transversal & Gestantes & $12,8^{(a)}$ \\
\hline \multirow[t]{2}{*}{$2004^{(34)}$} & São Paulo, SP & Longitudinal & Gestantes sem HIV & $16,7^{\text {(c) }}$ \\
\hline & & & Puérperas sem HIV & $25,0^{(\mathrm{c})}$ \\
\hline $2006^{(26)}$ & Rio de Janeiro, RJ & Transversal & Puérperas & $22,0^{(a)}$ \\
\hline $2006^{(35)}$ & Recife, PE & Transversal & Puérperas & $25,0^{(\mathrm{c})}$ \\
\hline
\end{tabular}

${ }^{(a)}$ retinol sérico $<1,05 \mu \mathrm{mol} / \mathrm{L}$; ${ }^{\left({ }^{b}\right)}$ retinol sérico $<0,70 \mu \mathrm{mol} / \mathrm{L}$; ${ }^{(\mathrm{c})}$ retinol sérico $<20,0 \mu \mathrm{g} / \mathrm{dL}$ (equivale $\mathrm{a}<0,70 \mu \mathrm{mol} / \mathrm{L}$ ).

Tabela 2 - Prevalência da deficiência de vitamina A em recém-nascidos, pré-escolares, escolares e adolescentes, em diferentes regiões do Brasil

\begin{tabular}{|c|c|c|c|c|}
\hline Ano & Localidade & Tipo de estudo & $\begin{array}{c}\text { Grupo populacional/ } \\
\text { Faixa Etária }\end{array}$ & Prevalência (\%) \\
\hline \multirow{2}{*}{$1995^{(36)}$} & \multirow{2}{*}{ SP - outras regiões } & \multirow[t]{2}{*}{ Caso-controle } & Recém-nascido com RCIU & $33,1^{(b)}$ \\
\hline & & & Recém-nascido adequado & $14,9^{(b)}$ \\
\hline $1995^{(37)}$ & SP - outras regiões & Transversal & 3-7 anos & $17,6^{(\mathrm{c})}$ \\
\hline $1995^{(38)}$ & Bahia - semi-árido & Transversal & 6-72 meses & $44,7^{(b)}$ \\
\hline $1996^{(39)}$ & Bahia - semi-árido & Transversal & Pré-escolar & $15,3^{(\mathrm{c})}$ \\
\hline $1998^{(13)}$ & Rio de Janeiro, RJ & Transversal & Recém-nascido & $55,7^{(a)}$ \\
\hline \multirow[t]{3}{*}{$2000^{(40)}$} & Manaus & \multirow[t]{3}{*}{ Transversal } & \multirow[t]{3}{*}{ Pré-escolar } & $15,5^{(\mathrm{e})}$ \\
\hline & Boa Vista & & & $32,4^{(e)}$ \\
\hline & Porto Velho & & & $32,4^{(e)}$ \\
\hline $2001^{(41)}$ & SP - fora da Capital & Transversal & Recém-nascido & $23,0^{(b)}$ \\
\hline $2001^{(14)}$ & Rio de Janeiro, RJ & Transversal & Pré-escolar & $34,6^{(a)}$ \\
\hline $2001^{(42)}$ & MG - fora da capital & Transversal & Pré-escolar & $15,0^{(b)}$ \\
\hline $2002^{(43)}$ & Brasília & Transversal & Pré-escolar & $60,6^{(\mathrm{e})}$ \\
\hline $2004 ; 2005^{(11,12)}$ & Rio de Janeiro, RJ & Transversal & Recém-nascido & $45,5^{(\mathrm{a})}$ \\
\hline $2004^{(34)}$ & São Paulo, SP & Longitudinal & Recém-nascido & $25,0^{(b)}$ \\
\hline $2005^{(4)}$ & SP - fora da capital & Transversal & 24-71 meses & $75,4^{(\mathrm{d})}$ \\
\hline $2005^{(24)}$ & MG - fora da capital & Transversal & 6-14 anos & $29,0^{(\mathrm{e})}$ \\
\hline \multirow[t]{2}{*}{$2005^{(45)}$} & \multirow[t]{2}{*}{ Recife, $\mathrm{PE}$} & \multirow[t]{2}{*}{ Transversal } & \multirow[t]{2}{*}{$6-59$ meses } & $22,0^{(a)}$ \\
\hline & & & & $7,0^{(b)}$ \\
\hline $2006^{(26)}$ & Rio de Janeiro, RJ & Transversal & Recém-nascido & $54,2^{(a)}$ \\
\hline \multirow[t]{3}{*}{$2007^{(23)}$} & \multirow[t]{3}{*}{ Rio de Janeiro, RJ } & \multirow[t]{3}{*}{ Transversal } & 7-17 anos & $10,0^{(f)}$ \\
\hline & & & 7-10 anos & $6,8^{(f)}$ \\
\hline & & & 10-17 anos & $12,6^{(\mathrm{f})}$ \\
\hline $2007^{(25)}$ & Brasília - regiões & Transversal & 5-18 anos & $34,0^{(b)}$ \\
\hline $2007^{(46)}$ & Teresina, $\mathrm{PI}$ & Transversal & $36-83$ meses & $15,4^{(b)}$ \\
\hline
\end{tabular}

RCIU: restrição do de crescimento intra-uterino; (a) retinol sérico $<1,05 \mu \mathrm{mol} / \mathrm{L}$; (b)retinol sérico $<0,70 \mu \mathrm{mol} / \mathrm{L}$; (c) retinol sérico $<0,35 \mu \mathrm{mol} / \mathrm{L}$; (d)teste de resposta sérica $>20,0 \%$ (equivalente a $<0,7 \mu \mathrm{mol} / \mathrm{L}$ ); ${ }^{\left({ }^{(e)}\right.}$ retinol sérico $<20,0 \mu \mathrm{g} / \mathrm{dL}$ (equivalente $\mathrm{a}<0,7 \mu \mathrm{mol} / \mathrm{L}$ ); ${ }^{\left({ }^{(}\right)} \mathrm{retinol}$ sérico $<30,0 \mu \mathrm{g} / \mathrm{dL}(\mathrm{equiva-}$ lente $\mathrm{a}<1,05 \mu \mathrm{mol} / \mathrm{L})$.

\section{Discussão}

O desenho adotado na maioria dos estudos analisados foi transversal. Porém, modelos de intervenção que viabilizem o diagnóstico e tratamento da carência por meio de estratégias de combate em médio e longo prazo, devem ser estimulados.
Os segmentos populacionais compostos por recémnascidos, gestantes, puérperas e pré-escolares constituem grupos tradicionalmente reconhecidos como de risco para a deficiência da vitamina $A$, devido ao momento biológico de extrema demanda nutricional, em fase de expressivo crescimento ou desenvolvimento. Há consenso universal de que 
crianças menores de seis anos pertencem ao grupo de maior risco para desenvolver manifestações clínicas da carência, já que suas necessidades de vitamina $A$ são proporcionalmente maiores que as de qualquer outro grupo etário devido ao seu rápido crescimento, além da maior prevalência de doenças infecciosas nessa faixa etária ${ }^{(17,19,20)}$.

Por outro lado, a literatura vem apontando outros grupos com potencial de risco para a deficiência da vitamina A, como os escolares e, especialmente, os adolescentes ${ }^{(20-22)}$. Na seleção dos artigos analisados, apenas Ramalho et al ${ }^{(15)}$, Da Silva $e t$ $a l^{(23)}$, Santos $e t a l^{(24)}$ e Graebner $e t$ al ${ }^{(25)}$ incluíram escolares e adolescentes em suas casuísticas, demonstrando que, no Brasil, a deficiência da vitamina A nesses grupos etários ainda é pouco estudada. Além disso, com o crescente aumento da obesidade na infância e adolescência e sua relação com a redução dos níveis sérico de vitamina $\mathrm{A}$, deve-se discutir e avaliar o estado nutricional antropométrico como variável independente para a deficiência da vitamina $\mathrm{A}^{(16)}$. Da Silva et al ${ }^{(23)}$ encontraram grande risco de níveis mais baixos de carotenóides em escolares e adolescentes com sobrepeso (OR 2,51; IC95\% 1,43-4,39). Diante disso, a literatura é unânime ao afirmar que a deficiência da vitamina $A$, assim como carências de outros micronutrientes, está mais associada ao consumo inadequado de alimentos fontes do que à classificação do estado nutricional antropométrico ${ }^{(16,26)}$.

Dentre os indicadores utilizados para o diagnóstico da deficiência da vitamina $\mathrm{A}$, o bioquímico é de grande importância e amplamente empregado em estudos epidemiológicos. A dosagem de retinol sérico é o método bioquímico mais adotado para avaliar o estado nutricional de vitamina A, sendo geralmente utilizado no cálculo das estimativas mundiais ${ }^{(1-3)}$. Além disso, tem sido empregado na validação de outros indicadores do estado nutricional de vitamina $\mathrm{A}$, tais como o funcional, o dietético e o leite humano ${ }^{(9,11,12,17,20,27)}$. Dentre os métodos de quantificação dos níveis séricos de retinol, citam-se aqueles usados para avaliação indireta das reservas hepáticas de vitamina A como resposta relativa a uma dose (RDR), resposta modificada relativa a uma dose (MRDR) e resposta sérica 30 dias após uma dose maciça de vitamina A $(+ \text { S30DR })^{(2)}$.

Em 1991 foi demonstrado que menos de 5\% das crianças brasileiras de baixo nível socioeconômico e com estado nutricional de vitamina $A$ adequado apresentaram níveis de retinol sérico abaixo de $1,05 \mu \mathrm{mol} / \mathrm{L}^{(28)}$. A partir de análise do National Health and Nutrition Examinations Survey, realizado nos Estados Unidos entre 1988 e $1994^{(29)}$, verificou-se que níveis séricos de retinol $<1,05 \mu \mathrm{mol} / \mathrm{L}$ foram mais prevalentes em crianças de quatro a 13 anos, sendo considerados como estado nutricional de vitamina A sub-ótimo, pois houve aumento dos níveis séricos de retinol após a suplementação com vitamina $\mathrm{A}^{(29)}$.

A Organização Mundial da Saúde (OMS), em 1996, passou a reconhecer que novas evidências científicas sugerem risco maior de mortalidade em populações sem sinais clínicos da carência ${ }^{(3)}$. Isso assinalou a necessidade de reavaliar pontos de corte e critérios para interpretar os níveis séricos de retinol, o que possibilitaria a detecção dos casos de deficiência ainda em estágio subclínico ou marginal, usando-se o ponto de corte $<1,05 \mu \mathrm{mol} / \mathrm{L}$. Tal valor está sendo aplicado com freqüência crescente para definir a deficiência da vitamina A em gestantes, puérperas, recém-nascidos, pré-escolares e escolares $^{(3,10,13,14)}$. Ressalta-se que esse ponto de corte já foi inter-validado com o teste terapêutico (+S30DR) e com o $\mathrm{RDR}^{(28)}$, além de apresentar associação com alterações funcionais da visão ${ }^{(11,12,27,30,31)}$. O International Vitamin A Consultive Group também registrou diversos estudos que reforçam a utilização do referido ponto de corte para diagnosticar a deficiência da vitamina $\mathrm{A}$ em variados grupos populacionais ${ }^{(32)}$. Com isso, a adoção de níveis séricos de retinol $<0,70 \mu \mathrm{mol} / \mathrm{L}$ definir deficiência da vitamina A permite questionar a possível subnotificação desse diagnóstico, o que conduz a uma reflexão quanto às atuais cifras de prevalências em nível populacional, que podem ser ainda maiores do que as atualmente relatadas no país.

Em todos os trabalhos identificados e analisados, a deficiência da vitamina A apresenta nível expressivo, como pode ser constatado nas Tabelas 1 e 2. Diante do número de indivíduos acometidos por essa deficiência em São Paulo e no Rio de Janeiro, a exemplo de outras regiões fora do eixo tradicional da miséria no Brasil, ainda que a forma mais prevalente seja a subclínica, confirma-se a hipótese de que essa carência nutricional não seja um problema restrito apenas às regiões de extrema pobreza do país. Observa-se, ainda, discrepância das tendências epidemiológicas descritas quanto ao declínio da desnutrição energética e protéica no Brasil $^{(47)}$.

Os estudos realizados em diferentes partes do país têm sido eficazes na medida em que identificam os grupos mais vulneráveis à carência de micronutrientes ${ }^{(9,48,49)}$, não havendo diferenças marcantes na ocorrência desta deficiência entre as diversas regiões. Isso significa que a deficiência não está restrita às camadas da população menos favorecidas, indicando haver uma história independente em relação ao curso histórico da desnutrição energética e protéica. Assim, 
pode-se sugerir que as medidas de intervenção para prevenir e combater a deficiência da vitamina A no país não devem ser limitadas às áreas do território brasileiro tradicionalmente incluídas no mapa de regiões endêmicas da carência.

Recentes achados obtidos em estudos intervencionais sobre suplementação de vitamina A têm sido animadores, apontando os benefícios decorrentes do atendimento às necessidades de vitamina A e constituição da reserva hepática no grupo materno-infantil, em especial para aqueles indivíduos com inadequação nutricional desta vitamina ${ }^{(50)}$.

No Brasil, ainda não existe um estudo formal sobre a avaliação do impacto da suplementação de vitamina A em puérperas, lactentes e pré-escolares, mas a meta para cobertura pelo Programa Vitamina A Mais é de $75 \%$ para crianças de 6 a 11 meses, $100 \%$ (primeria dose) e 60\% (segunda dose) para crianças de 12 a 59 meses, e $60 \%$ das puérperas, nas regiões consideradas endêmicas para deficiência da vitamina $A^{(51)}$. Dados recentes sobre a cobertura do programa 'Vitamina A Mais' mostram que a mesma foi de $63 \%$ para crianças entre 6 e 11 meses; $35 \%$ e $16 \%$ para primeira e segunda doses em crianças de 12 a 59 meses, respectivamente. Com relação às puérpera no pós-parto imediato, os dados mais recentes disponíveis sobre a cobertura são referentes ao ano de $2006(70 \%)^{(51)}$.

O panorama da deficiência da vitamina A em gestantes e puérperas, segundo o indicador bioquímico nas diferentes regiões do Brasil no período estudado, vai ao encontro das estimativas dos Comitês de Saúde e Micronutrientes, que apontam o país como uma área de alta prevalência da deficiência. Tais comitês ressaltam a importância da investigação desta deficiência durante a gestação, assim como a viabilização de ações de prevenção e controle da morbimortalidade no grupo materno-infantil ${ }^{(3,7,8)}$.

Considerando-se os períodos fisiológicos de grande vulnerabilidade, (gestação e lactação), a deficiência da vitamina A está relacionada à prematuridade, a síndromes hipertensivas da gestação, à sepse puerperal, à anemia gestacional, ao estresse oxidativo e a uma maior chance de aborto espontâneo. Tudo isso contribui para o aumento das taxas de mortalidade materna e de lactentes nos primeiros meses de vida ${ }^{(17)}$. Nesse sentido, um assunto bastante discutido na literatura atual é a relação entre níveis séricos de vitamina $\mathrm{A}$ e a transmissão vertical da infecção em gestantes portadoras do vírus da imunodeficiência adquirida. Um dos artigos analisados abordou a associação entre vitamina $\mathrm{A}$ e resultados obstétricos e perinatais em gestantes portadoras do vírus da Aids. Porém, optou-se por apresentar os valores de prevalência referentes às mulheres do grupo controle do estudo, não portadoras do vírus, a fim de que não se criasse confusão na interpretação dos achados ${ }^{(34)}$.

O presente levantamento de dados mostra que as informações referentes à prevalência de deficiência de vitamina $\mathrm{A}$ em gestantes e puérperas ainda precisam ser mais bem documentadas no cenário nacional, sobretudo se comparadas aos dados de prevalência em pré-escolares disponíveis na literatura. Tal informação é de grande relevância e valor científico diante da necessidade de uma melhor compreensão da ação deste nutriente no resultado obstétrico, principalmente em estudos nacionais, para que este conhecimento seja disseminado na prática da assistência pré-natal.

Dados recentes de Ramalho et al ${ }^{(26)}$ apontam a falta de associação entre a deficiência de vitamina $\mathrm{A}$ e a condição sociodemográfica. Tal constatação aponta o baixo consumo de vitamina A como o principal fator etiológico em nível epidemiológico. A exclusão e o baixo consumo dessa vitamina estão mais relacionados a questões culturais e hábitos alimentares do que a fatores econômicos. $\mathrm{O}$ aumento do consumo de alimentos ricos em vitamina A é uma importante estratégia no combate e prevenção à deficiência de vitamina A no grupo materno-infantil.

A deficiência de vitamina $A$ e suas graves conseqüências para a Saúde Pública são impostas a uma parcela expressiva da população. Assim, medidas de consolidação efetiva do Programa Nacional de Suplementação de Vitamina A (Vitamina A Mais), estratégias de intervenção adicionais à suplementação, como a fortificação de alguns alimentos básicos e a promoção de mudança de hábitos alimentares com educação nutricional dirigida a grupos de risco, devem ser encorajadas em todo o território nacional, sem restrição a regiões específicas, tendo em vista os achados que demonstram a evolução do estado nutricional relativo à vitamina $\mathrm{A}$ no Brasil nos últimos anos.

Considerando-se a dimensão coletiva da deficiência de vitamina A refletida nas taxas de morbimortalidade do grupo materno-infantil, os resultados encontrados apontam para a inclusão de estratégias de intervenções adicionais à suplementação e fornecem subsídios para reavaliar os pontos de corte e critérios adotados na interpretação dos níveis séricos de retinol para o diagnóstico da deficiência. Essa análise crítica da literatura direciona a uma reflexão sobre a pertinência da inclusão de grupos com potencial risco para a deficiência em programas de intervenção, além de alertar para a necessidade de avaliação do impacto de ações direcionadas à prevenção e erradicação da deficiência de vitamina $\mathrm{A}$ no país e de ampliação de sua cobertura. 


\section{Referências bibliográficas}

1. Mason JB, Lotfi M, Dalmiya N, Sethuraman K, Deitchler M. The micronutrient report: current progress and trends in the control of vitamin $\mathrm{A}$, iodine, and iron deficiencies. Ottawa, Canada: The Micronutrient Iniciative/Unicef; 2001.

2. McLaren DS, Frigg M. Manual de ver y vivir sobre los transtornos por deficiencia de vitamina A (VADD). Washington: OPAS/OMS; 1999.

3. World Health Organization. Indicators for assessing vitamin A deficiency and their application in monitoring and evaluating intervention programmes. Geneva: WHO; 1996.

4. Ferraz IS, Daneluzzi JC, Vannucchi H, Jordão AA Jr, Ricco RG, Del Ciampo LA et al. Prevalence of iron deficiency and its association with vitamin A deficiency in preschool children. J Pediatr (Rio J) 2005;81:169-74.

5. World Health Organization. Reducción de la mortalidad materna. Declaración conjunta OMS/FNUAP/UNICEF/Banco Mundial. Genebra: OMS; 1999.

6. Martins MC, Oliveira YP, Coitinho DC, Santos LM. Panorama das ações de controle da deficiência de vitamina A no Brasil. Rev Nutr 2007;20:5-18.

7. Organización Panamericana de la Salud. Visión Integrada de la suplementación con vitamina A en las Américas: 2-4 de mayo del 2001; Managua, Nicaragua; informe de la reunión regional. Washington: OPAS; 2001.

8. Brasil - Secretaria de Atenção a Saúde. Vitamina A mais: programa nacional de suplementação de vitamina A - condutas gerais. Brasília: Ministério da Saúde, 2004

9. Brasil - Secretaria de Políticas de Saúde. Projeto suplementação de mega dose de Vitamina A no pós-parto imediato nas maternidades/hospitais. Brasília: Ministério da Saúde, 2002.

10. Accioly E, Souza Queiroz S. Deficiencia de vitamina A en embarazadas asistidas en una maternidad pública en Rio de Janeiro, Brasil. Rev Chilena Nutr 2000;27:316-9.

11. Saunders C, Leal MC, Gomes MM, Campos LF, Silva BA, Lima AP et al. Gestational night blindness in women treated at a public maternity hospital in Rio de Janeiro, Brazil. J Health Popul Nutr 2004;22:348-56.

12. Saunders C, Ramalho RA, Lima AP, Gomes MM, Campos LF, Silva BA et al. Association between gestational night blindness and serum retinol in mother/ newborn pairs in the city of Rio de Janeiro, Brazil. Nutrition 2005;21:456-61.

13. Ramalho RA, Anjos LA, Flores H. Hypovitaminosis A in neonates in 2 public maternity hospitals in Rio de Janeiro, Brazil. Cad Saude Publica 1998;14:821-7.

14. Ramalho RA, Anjos LA, Flores $H$. Valores séricos de vitamina A e teste terapêutico em pré-escolares atendidos em uma Unidade de Saúde do Rio de Janeiro, Brasil. Rev Nutr 2001;14:5-12.

15. Ramalho RA, Saunders C, Natalizi DA, Cardoso LO, Accioly E. Níveis séricos de retinol em escolares de 7 a 17 anos no município do Rio de Janeiro. Rev Nutr 2004;17:461-8.

16. Sarni RS, Kochi C, Ramalho RA, Schoeps DO, Sato K, Mattoso L et al. Impact of vitamin A megadose supplementation on the anthropometry of children and adolescents with non-hormonal statural deficit: a double-blind and randomized clinical study. Int J Vitam Nutr Res 2003;73:303-11.

17. Eigbefoh JO, Okpere EE, Ande B, Asonye C. How useful is the Helen Keller food frequency chart in the determination of the vitamin A status in pregnancy? J Obstet Gynaecol 2005;25:123-7.

18. Brasil - Secretaria de Atenção a Saúde: Caderneta da saúde da criança. $3^{\circ}$ ed. Brasília: Ministério da Saúde, 2007.

19. Saunders C, Ramalho RA, Leal MC. Estado nutricional de vitamina A no grupo materno-infantil. Rev Bras Saúde Matern Infant 2001;1:21-9.

20. Underwood BA. Vitamin A deficiency disorders: international efforts to control a preventable "Pox". J Nutr 2004;134:231S-6S.

21. Ahmed F, Rahman A, Noor AN, Akhtaruzzaman M, Hughes R. Anaemia and vitamin A status among adolescent schoolboys in Dhaka City, Bangladesh. Public Health Nutr 2006;9:345-50.

22. Lanerolle $P$, Atukorala $S$. Nutrition education improves serum retinol concentration among adolescent school girls. Asia Pac J Clin Nutr 2006;15:43-9.

23. de Souza Valente da Silva L, Valeria da Veiga G, Ramalho RA. Association of serum concentrations of retinol and carotenoids with overweight in children and adolescents. Nutrition 2007;23:392-7.
24. Santos MA, Rezende EG, Lamounier JA, Galvão MA, Bonomo E, Leite RC Hipovitaminose A em escolares da zona rural de Minas Gerais. Rev Nutr 2005;18:331-9.

25. Graebner IT, Saito CH, de Souza EM. Biochemical assessment of vitamin A in schoolchildren from a rural community. J Pediatr (Rio J) 2007;83:247-52.

26. Ramalho RA, Flores H, Accioly E, Saunders C. Associação entre deficiência de vitamina $A$ e situação sociodemográfica de mães e recém-nascidos. Rev Assoc Med Bras 2006;52:170-5

27. Dimenstein R, Simplício JL, Ribeiro KD, Melo IL. Retinol levels in human colostrum: influence of child, maternal and socioeconomic variables. J Pediatr (Rio J) 2003;79:513-8

28. Flores H, Azevedo MN, Campos FA, Barreto-Lins MC, CavalcantiAA, Salzano AC et al. Serum vitamin A distribution curve for children aged 2-6 y known to have adequate vitamin A status: a reference population. Am J Clin Nutr 1991;54:707-11.

29. Ballew C, Bowman BA, Sowell AL, Gillespie C. Serum retinol distributions in residents of the United States: third National Health and Nutrition Examination Survey, 1988-1994. Am J Clin Nutr 2001;73:586-93.

30. Christian P, West KP Jr, Khatry SK, Katz J, Shrestha SR, Pradhan EK et al. Night blindness of pregnancy in rural Nepal-nutritional and health risks. Int $\mathrm{J}$ Epidemiol 1998;27:231-7.

31. Sommer A, Davidson FR. Assessment and control of vitamin A deficiency: the Annecy Accords. J Nutr 2002;132 (Suppl 9):2845S-50.

32. International Vitamin A Consultive Group. Report of the XXI international vitamin A consultative group meeting, Marrakech, Morocco, February 2003. Washington, DC: IVACG; 2003.

33. Rondó PH, Villar BS, Tomkins AM. Vitamin A status of pregnant women assessed by a biochemical indicator and a simplified food frequency questionnaire. Arch Latinoam Nutr 1999;49:322-5.

34. El Beitune P, Duarte G, Vannucchi H, Quintana SM, Figueiro-Filho EA, de Morais EN, et al. Serum vitamin A during pregnancy and effects on obstetrics and perinatal outcomes in HIV infected pregnant women. Arch Latinoam Nutr 2004; $54: 419-27$

35. Lopes RE, Ramos KS, Bressani CC, Arruda IK, Souza Al. Prevalência de anemia e hipovitaminose $\mathrm{A}$ em puérperas do Centro de Atenção à Mulher do Instituto Materno Infantil Prof Fernando Figueira, IMIP: um estudo piloto. Rev Bras Saude Matern Infant 2006;6 (Suppl 1):S63-8.

36. Rondo PH, Abbott R, Rodrigues LC, Tomkins AM. Vitamin A, folate and iron concentration in cord and maternal blood of intra-uterine growth retarded and appropriate birth weight babies. Eur J Clin Nutr 1995;49:391-9.

37. Gonçalves-Carvalho CM, Amaya-Farfan J, Wilke BC, Vencovsky R. Prevalence of hypovitaminosis a in children of peripheral districts of Campinas São Paulo, Brazil. Cad Saude Publica 1995;11:85-96.

38. Prado MS, Assis AM, Martins MC, Nazaré MP, Rezende IF, Conceição ME. Vitamin A deficiency in children of rural zones, Northeast region of Brazil. Rev Saude Publica 1995;29:295-300.

39. Santos LM, Assis AM, Martins MC, Araújo MP, Morris SS, Barreto ML. Situação nutricional e alimentar de pré-escolares no semi-árido da Bahia (Brasil): ॥ hipovitaminose A. Rev Saude Publica 1996;30:67-74.

40. Marinho HA. Prevalência de deficiência de vitamina A em pré-escolares de três capitais da Amazônia Ocidental Brasileira [tese de doutorado]. São Paulo (SP): USP; 2000.

41. Rondó PH, Abbott R, Tomkins AM. Vitamin A and neonatal anthropometry. J Trop Pediatr 2001;:47:307-10.

42. Magalhães $P$, Ramalho RA, Colli $C$. Deficiência de ferro e de vitamina $A$ avaliação nutricional de pré-escolares de Viçosa, MG, Brasil. Nutrire Rev Soc Bras Aliment Nutr 2001;21:41-56.

43. Muniz-Junqueira MI, Queiroz EF. Relação entre desnutrição energéticoprotéica, vitamina A, e parasitoses em crianças vivendo em Brasília. Rev Soc Bras Med Trop 2002;35:133-42.

44. Martins MC, Santos LM, Assis AM. Prevalence of hypovitaminosis A among preschool children from northeastern Brazil, 1998. Rev Saude Publica 2004;38:537-42. 
45. Fernandes TF, Diniz AS, Cabral PC, Oliveira RS, Lola MM, Silva SM et al. Hipovitaminose A em pré-escolares de creches públicas do Recife: indicadores bioquímico e dietético. Rev Nutr 2005;18:471-80.

46. PaivaAA, Rondó PH, Gonçalves-Carvalho CM, Illison VK, Pereira JA, Vaz-de-Lima LR et al. Prevalence and factors associated with vitamin A deficiency in preschool children from Teresina, Piauí, Brazil. Cad Saude Publica 2006;22:1979-87.

47. Batista Filho M, Rissin A. Nutritional transition in Brazil: geographic and temporal trends. Cad Saúde Pública 2003;19 (Suppl 1):S181-91.

48. Ramalho RA, Flores $\mathrm{H}$, Saunders $\mathrm{C}$. Hipovitaminose A no Brasil: um problema de saúde pública. Rev Panam Salud Publica 2002;12:117-22.
49. Sommer A, Hussaini G, Muhilal, Tarwotjo I, Susanto D, Saroso S. History of nightblindness: a simple tool for xerophthalmia screening. Am J Clin Nutr 1980;33:887-91.

50. Schémann JF, Banou A, Malvy D, Guindo A, Traore L, Momo G. National immunisation days and vitamin A distribution in Mali: has the vitamin A status of pre-school children improved? Public Health Nutr 2003;6:233-40.

51. Brasil - Secretaria de Atenção a Saúde [homepage on the Internet] Vitamina A mais: programa nacional de suplementação de vitamina A [cited 2007 Nov 07]. Available from: http://dtr2004.saude.gov.br/nutricao/ vita_relatorio.php 